\title{
R-MPV followed by high-dose chemotherapy with thiotepa-based and autologous stem cell transplantation for newly diagnosed primary central nervous system lymphoma: a single-center experience
}

\author{
Ji Yun Lee ${ }^{1}$, Jin Ho Paik ${ }^{2}$, Koung Jin Suh ${ }^{1}$, Ji-Won Kim ${ }^{1}$, Se Hyun Kim ${ }^{1}$, Jin Won Kim ${ }^{1}$, Yu Jung Kim ${ }^{1}$, \\ Keun-Wook Lee ${ }^{1}$, Jee Hyun Kim ${ }^{1}$, Soo-Mee Bang ${ }^{1}$, Jong-Seok Lee ${ }^{1}$, Jeong-Ok Lee ${ }^{1}$ \\ ${ }^{1}$ Department of Internal Medicine, ${ }^{2}$ Department of Pathology, Seoul National University Bundang Hospital, Seongnam, Korea
}

p-ISSN 2287-979X / e-ISSN 2288-0011 https://doi.org/10.5045/br.2021.2021073 Blood Res 2021;56:285-292.

Received on April 12, 2021 Revised on August 27, 2021 Accepted on October 18, 2021

\author{
Correspondence to \\ Jeong-Ok Lee, M.D., Ph.D. \\ Division of Hematology and Medical \\ Oncology, Department of Internal \\ Medicine, Seoul National University \\ Bundang Hospital, Seoul National \\ University College of Medicine, 82 \\ Gumi-ro, 173 Beon-gil, Bundang-gu, \\ Seongnam 13620, Korea \\ E-mail: jeongok77@gmail.com \\ (C) 2021 Korean Society of Hematology
}

\section{Background}

High-dose chemotherapy followed by autologous stem cell transplantation (HDC-ASCT) as a consolidation treatment is a promising approach for eligible patients with newly diagnosed primary central nervous system lymphoma (PCNSL).

Methods

In this retrospective analysis, 22 patients with newly diagnosed PCNSL received chemotherapy with rituximab, methotrexate, procarbazine, and vincristine. Those who showed complete or partial response subsequently received consolidation HDC-ASCT with a thiotepa-based conditioning regimen but did not undergo radiotherapy.

\section{Results}

The PCNSL patients had a median age of 57 years (range, 49-67 yr); of the total patients, $9.1 \%$ had a performance status of 2 or higher, and $72.1 \%$ had multiple lesions. Approximately $82 \%$ of patients received six cycles of induction chemotherapy, which was well tolerated with excellent disease control. The rate of confirmed or unconfirmed complete response increased from $45.5 \%$ at the period of interim analysis to $81.8 \%$ prior to the initiation of HDC-ASCT. With a median follow-up of 19.6 months (range, 7.5-56.5 $\mathrm{mo}$ ), the 2-year progression-free survival and overall survival estimates were $84 \%$ and $88 \%$, respectively. No treatment-related deaths occurred. Grade 3 toxicity was recorded in $90.9 \%$ of the patients after undergoing the HDC-ASCT, and the most common grade 3 adverse event was febrile neutropenia without sepsis.

\section{Conclusion}

The discussed treatment approach is feasible in patients with newly diagnosed PCNSL, yielding encouraging results.

Key Words Primary CNS lymphoma, Autologous stem-cell transplantation, Consolidation, Thiotepa

\section{INTRODUCTION}

Primary central nervous system lymphoma (PCNSL) is an aggressive form of extranodal non-Hodgkin lymphoma that involves the brain, meninges, eyes, and/or spinal cord without systemic involvement [1]. PCNSL accounts for 3\% to $4 \%$ of all primary brain tumors and $4 \%$ to $6 \%$ of extranodal lymphomas [2].
First-line induction chemotherapy regimens incorporating high-dose methotrexate (HD-MTX) are considered the standard of care for patients with newly diagnosed PCNSL [3]. HD-MTX is commonly used in combination with other drugs such as vincristine, procarbazine, cytarabine, rituximab, and temozolomide, resulting in high rates of initial response (6995\%) [4-6]. Although PCNSL is sensitive to chemotherapy, more than half of patients who show complete response (CR) will experience relapse within 5 years [7]. Consolidative

(2) This is an Open Access article distributed under the terms of the Creative Commons Attribution Non-Commercial License (http://creativecommons.org/licenses/by-nc/4.0) 6 which permits unrestricted non-commercial use, distribution, and reproduction in any medium, provided the original work is properly cited. 
whole-brain radiotherapy (WBRT) is the most commonly adopted treatment strategy for prolonging remission after induction therapy [8]. However, major concerns regarding neurotoxicity, which can have a tremendous impact on the quality of life of long-term survivors, have prompted the search for alternative consolidative strategies [9].

Previous retrospective and phase II trials have suggested the feasibility and efficacy of high-dose chemotherapy followed by autologous stem-cell transplantation (HDC-ASCT) as a consolidation treatment for patients with PCNSL [10-15]. Most trials addressing HDC-ASCT have included only a small number of patients with a short follow-up period and have demonstrated differences in clinical features and conditioning regimens. In addition, a proportion of patients in these studies also received WBRT as an adjuvant treatment post-ASCT to all patients or as induction therapy to patients who did not achieve a CR. Thus, it is difficult to determine the efficacy and feasibility of HDC-ASCT based on these previous data.

A recent phase II trial of a combination of rituximab, methotrexate, procarbazine, and vincristine (R-MPV) followed by HDC-ASCT with a thiotepa, busulfan, and cyclophosphamide (TBC) conditioning regimen reported excellent disease control, a 2-year progression-free survival (PFS) rate of $79 \%$, and acceptable toxicity profiles with minimal neurotoxicity in patients with newly diagnosed PCNSL [16]. This retrospective study aimed to further investigate the use of R-MPV followed by thiotepa-based HDC-ASCT as consolidation treatment in patients with newly diagnosed PCNSL.

\section{MATERIALS AND METHODS}

\section{Patients}

A total of 22 patients with newly diagnosed PCNSL underwent thiotepa-based HDC-ASCT as consolidation therapy at Seoul National University Bundang Hospital between January 2015 and December 2019. All patients had a biopsy-proven diagnosis of PCNSL with diffuse large B-cell lymphoma, except for one patient at risk of postoperative neurologic deficit. The patients' baseline characteristics obtained for analysis were age, sex, Eastern Cooperative Oncology Group performance status (ECOG PS), lactate dehydrogenase (LDH) levels, cerebrospinal fluid (CSF) protein concentration, and CSF cytological results. The clinical prognostic factors were assessed using the International Extranodal Lymphoma Study Group (IELSG) scoring system [17].

The study was carried out in accordance with the Declaration of Helsinki and was approved by the Institutional Review Board (IRB) of Bundang Hospital. The need for patient consent was waived by the IRB due to the retrospective nature of the study.

\section{Treatment and response evaluation}

All patients received induction chemotherapy with R-MPV, which was administered as follows: day $1,375 \mathrm{mg} / \mathrm{m}^{2}$ of rituximab; day $2,3.0 \mathrm{~g} / \mathrm{m}^{2}$ of methotrexate (over $3 \mathrm{hr}$ ), $1.4 \mathrm{mg} / \mathrm{m}^{2}$ of vincristine; and days $1-7,100 \mathrm{mg} / \mathrm{m}^{2} / \mathrm{d}$ of procarbazine (odd cycles only). Hydration therapy and leucovorin rescue were administered according to institutional guidelines. Patients with evidence of malignancy in the CSF received $15 \mathrm{mg}$ of intrathecal methotrexate between cycles. Patients with CR, unconfirmed $\mathrm{CR}(\mathrm{uCR})$, or partial response (PR) directly underwent HDC-ASCT.

Meanwhile, patients received busulfan/thiotepa $(\mathrm{Bu} / \mathrm{TT})$ or TBC separately as a conditioning chemotherapy regimen. The $\mathrm{Bu} / \mathrm{TT}$ group received busulfan $(3.2 \mathrm{mg} / \mathrm{kg}$ intravenously) from day 8 (eight days prior to the initiation of stem cell infusion) to day 5 , thiotepa ( $5 \mathrm{mg} / \mathrm{kg}$ intravenously) from day 4 to day 3 , and stem cell infusion on day 0 . The TBC group received thiotepa $\left(250 \mathrm{mg} / \mathrm{m}^{2}\right.$ intravenously) from day 9 to day 7 , busulfan ( $3.2 \mathrm{mg} / \mathrm{kg}$ intravenously) from day 6 to day 4 , cyclophosphamide $(60 \mathrm{mg} / \mathrm{kg}$ intravenously) from day 3 to day 2, and stem cell infusion on day 0. G-CSF was administered daily from day 1 of ASCT until the neutrophil count was $<3,000$ cells $/ \mu L$. Neutrophil engraftment and platelet engraftment were defined as an absolute neutrophil count of $>0.5 \times 10^{9} / \mathrm{L}$ in the first three consecutive days and a platelet count of $>20 \times 10^{9} / \mathrm{L}$ without transfusion support, respectively. Patients $>60$ years old or less fit patients based on the clinical judgment of the physician were treated with low-intensity $\mathrm{TBC}$ or $\mathrm{Bu} / \mathrm{TT}$ regimen (Supplementary Table 1).

Response to treatment was assessed using contrast-enhanced brain magnetic resonance imaging (MRI), which was performed after two or three cycles of chemotherapy and before undergoing HDT-ASCT. Treatment response was defined based on the changes in the size of enhanced lesions on T1-weighted MRI and following the National Cancer Institute standardized response criteria [18]. The severity of adverse events was graded based on the National Cancer Institute Common Terminology Criteria for Adverse Events, version 4.0 .

\section{Statistical analysis}

The primary outcome measures were response rate, PFS, and overall survival (OS) after diagnosis. OS was defined as the time from initiation of the induction regimen to death from any cause, while PFS was defined as the time from initiation of the induction regimen to treatment failure or death from any cause. The survival curves were estimated using the Kaplan-Meier method and compared using the log-rank test. Univariate analyses were performed to identify the prognostic factors for PFS and OS using a Cox proportional hazards model. A chi-square test was conducted to compare the variables between the two groups as appropriate. All tests were two tailed, and a $P$-value of $<0.05$ was considered significant. All statistical analyses were performed using the Statistical Package for the Social Sciences version 18.0 (SPSS, Chicago, IL, USA). 


\section{RESULTS}

\section{Patients' characteristics}

The median age of the study group was 57 years (range, 59-67 yr), and $31.8 \%$ of the patients were $>60$ years old. In addition, $59.1 \%$ of the patients were men. At baseline, 9.1\% of our cohort had an ECOG PS of grade 2 or above, 26.3\% had an elevated LDH level, and $95.5 \%$ showed an elevated CSF protein concentration. Among the 19 patients in whom complete IELSG PCNSL prognostic parameters were available, 17 (89.5\%) presented an intermediate-to-high level of risk. Two patients $(9.1 \%)$ had a positive CSF cytological result, $13.6 \%$ had intraocular lymphoma, and $72.7 \%$ had multiple brain lesions. None of the patients had evidence of systemic lymphoma at the time of enrollment (Table 1).

Table 1. Patient's baseline characteristics $(N=22)$.

\begin{tabular}{|c|c|c|}
\hline Characteristics & $\mathrm{N}$ & $\%$ \\
\hline Age, median years (range) & \multicolumn{2}{|c|}{$57(49-67)$} \\
\hline Age $>60$ years & 7 & 31.8 \\
\hline Male sex & 13 & 59.1 \\
\hline ECOG PS $\geq 2$ & 2 & 9.1 \\
\hline Elevated $\mathrm{LDH}^{\mathrm{a})}$ & 5 & 26.3 \\
\hline Elevated CSF protein ${ }^{\text {b) }}$ & 21 & 95.5 \\
\hline Deep brain lesions & 16 & 72.7 \\
\hline \multicolumn{3}{|l|}{ IELSG risk group $^{a)}$} \\
\hline Low & 2 & 10.5 \\
\hline Intermediate & 14 & 73.7 \\
\hline High & 3 & 15.8 \\
\hline Positive CSF cytology & 2 & 9.1 \\
\hline \multicolumn{3}{|l|}{ Ocular involvement } \\
\hline Positive or suspicious & 3 & 13.6 \\
\hline Negative & 16 & 72.7 \\
\hline Unknown & 3 & 13.6 \\
\hline Multiple lesions & 16 & 72.7 \\
\hline
\end{tabular}

${ }^{a}$ Data regarding serum LDH and IELGS risk groups were available in 19 patients. ${ }^{\mathrm{b})}$ The cutoff values for normal CSF protein concentration were $45 \mathrm{mg} / \mathrm{dL}$ in patients $\leq 60$ years old and $60 \mathrm{mg} / \mathrm{dL}$ in patients older than 60 years.

Abbreviations: CSF, cerebrospinal fluid; ECOG PS, Eastern

Cooperative Oncology Group performance status; IELSG, international extranodal lymphoma study group; LDH, lactate dehydrogenase.

\section{Induction chemotherapy}

A majority of patients (18 patients, $81.8 \%$ ) received six cycles, three patients (13.6\%) received five cycles, and one patient $(4.5 \%)$ received eight cycles of induction chemotherapy. The details of the treatment responses are outlined in Table 2. During interim analysis after induction chemotherapy, 10 patients $(45.5 \%)$ achieved $\mathrm{CR} / \mathrm{CRu}$, while 12 patients (54.5\%) achieved PR. At the end of induction chemotherapy, eight of the patients who had previously achieved PR ultimately achieved CR/CRu. Therefore, after completion of induction chemotherapy, 18 patients (81.8\%) achieved $\mathrm{CR} / \mathrm{CRu}$, while 4 patients (18.2\%) achieved PR.

Induction chemotherapy was well tolerated with no reports of treatment-related deaths or treatment discontinuation due to toxicity. Grade 3 and 4 toxicities were noted in $50.5 \%$ and $4.5 \%$ of patients, respectively. Nine patients (40.9\%) developed grade 3 neutropenia, but febrile neutropenia did not occur. Four patients (18.2\%) experienced grade 3 hepatic impairment, but their liver enzyme levels returned to baseline values with daily liver function monitoring and receiving hydration therapy. One patient demonstrated grade 4 hepatic impairment after cycle 1 ; rituximab was omitted during subsequent chemotherapy cycles as it was the only drug that the patient received immediately after experiencing deterioration in liver function.

\section{High-dose chemotherapy and autologous stem cell transplantation}

The characteristics of the HDC-ASCT treatments are summarized in Table 3. All patients underwent therapy with TBC $(\mathrm{N}=12,54.5 \%)$ or $\mathrm{Bu} / \mathrm{TT}(\mathrm{N}=10,45.5 \%)$ as a conditioning regimen followed by ASCT. The median number of infused CD34 ${ }^{+}$cells was $6.7 \times 10^{6}$ cells $/ \mathrm{kg}$ (range, $4.1-21.1 \times 10^{6}$ cells $/ \mathrm{kg}$ ). The median number of days from treatment to neutrophil engraftment and platelet recovery was 9 days (range, 7-11 days) and 10 days (range, 7-12 days), respectively. The median length of transplantation-related hospitalization was 21 days (range, 18-44 days).

No treatment-related mortality (TRM) was reported. Grade 3 toxicities were observed in $90.9 \%$ of the patients, while none of the patients developed grade 4 toxicities. The most common grade 3 adverse events were febrile neutropenia $(86.4 \%)$, diarrhea (27.3\%), mucositis $(22.7 \%)$, colitis $(18.2 \%)$, and nausea (13.6\%). Of the 19 patients with febrile

Table 2. Treatment response.

\begin{tabular}{|c|c|c|c|c|c|c|c|c|}
\hline \multirow{2}{*}{ Response } & \multicolumn{2}{|c|}{ Induction_interim } & \multicolumn{2}{|c|}{ Pre-HDC-ASCT } & \multicolumn{2}{|c|}{ Post-HDC-ASCT } & \multicolumn{2}{|c|}{ Follow-up } \\
\hline & $\mathrm{N}$ & $\%$ & $\mathrm{~N}$ & $\%$ & $\mathrm{~N}$ & $\%$ & $N$ & $\%$ \\
\hline CR & 3 & 13.6 & 8 & 36.4 & 18 & 81.8 & 16 & 72.7 \\
\hline CRu & 7 & 31.8 & 10 & 45.4 & 4 & 18.2 & 2 & 9.1 \\
\hline PR & 12 & 54.5 & 4 & 18.2 & 0 & 0 & 0 & 0 \\
\hline PD & 0 & 0 & 0 & 0 & 0 & 0 & 4 & 18.2 \\
\hline
\end{tabular}

Abbreviations: CR, complete response; CRu, CR unconfirmed; HDC-ASCT, high-dose chemotherapy, and autologous stem cell transplant; PD, progressive disease; $\mathrm{PR}$, partial response. 
Table 3. HDC-ASCT characteristics.

\begin{tabular}{|c|c|c|}
\hline & $\mathrm{N}$ & $\%$ \\
\hline \multicolumn{3}{|l|}{ Conditioning regimen } \\
\hline TBC & 12 & 54.5 \\
\hline $\mathrm{Bu} / \mathrm{TT}$ & 10 & 45.5 \\
\hline $\begin{array}{l}\text { Number of infused CD34 }{ }^{+} \text {cells }\left(\times 10^{6} \text { cells } / \mathrm{kg}\right), \\
\text { median (range) }\end{array}$ & \multicolumn{2}{|c|}{$6.7(4.1-21.1)$} \\
\hline Neutrophil engraftment, median days (range) & \multicolumn{2}{|c|}{$9(7-11)$} \\
\hline Platelet engraftment, median days (range) & \multicolumn{2}{|c|}{$10(7-12)$} \\
\hline $\begin{array}{l}\text { Transplantation hospitalization, median days } \\
\text { (range) }\end{array}$ & \multicolumn{2}{|c|}{$21(18-44)$} \\
\hline
\end{tabular}

Abbreviations: Bu/TT, busulfan/thiotepa; TBC, thiotepa, busulfan, and cyclophosphamide.

neutropenia, 2 had bacteremia but eventually recovered after receiving appropriate antibiotic treatment without sepsis.

\section{PFS and OS}

The PFS and OS curves are shown in Fig. 1. After a median follow-up period of 19.6 months (range, 7.5-56.5 mo), 19 patients $(86.4 \%)$ survived and 17 patients $(77.3 \%)$ had no signs of disease progression. The median PFS and OS were 38.7 months [95\% confidence interval (CI), 23.6-49.6] and 43.5 months (95\% CI, 31.3-55.7), respectively. The 2-year PFS and OS rates were $84 \%$ and $88 \%$, respectively.

Four patients $(18.2 \%)$ had a tumor relapse in the CNS and received second-line treatment. One patient who experienced a relapse 20 months after transplantation survived after receiving several salvage therapies, while another patient who had a relapse 8 months after transplantation died from progressive lymphoma refractory due to salvage therapy. One patient is currently undergoing WBRT, and another patient died of pneumonia after receiving salvage WBRT. One patient died of aspiration pneumonia, with no evidence of disease recurrence.

\section{DISCUSSION}

In this retrospective study, patients with newly diagnosed PCNSL were treated with R-MPV induction chemotherapy, followed by consolidation HDC-ASCT with a thiotepa-based conditioning regimen in those who showed a response, but did not receive further treatment until disease progression.

With regard to the adapted treatment strategy, there are two major points that should be discussed. The first point is related to the efficacy of R-MPV therapy as induction regimen prior to the initiation of ASCT. Based on the results of retrospective studies comparing historical controls, addition of rituximab to HD-MTX-based chemotherapy improves the chance of CR and OS in patients with newly diagnosed PCNSL [19-21]. Recently, results from the first randomization of the IELSG 32 trial indicated that the addition of rituximab and thiotepa to conventional methotrexate-cytarabine combination therapy (known as the MATRix regi-

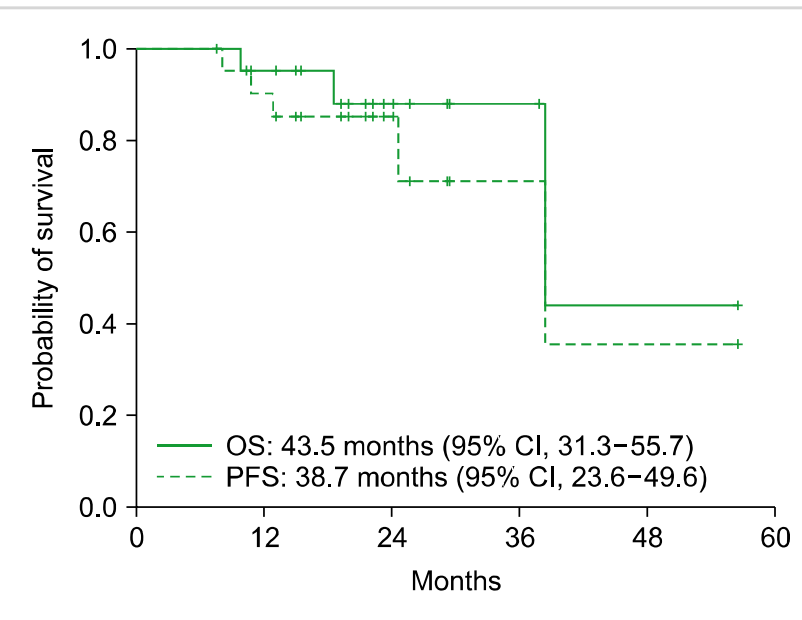

Fig. 1. Kaplan-Meier curves for OS and PFS.

men) was associated with an overall response rate (ORR) of $87 \%$ and a 2 -year PFS rate of $62 \%$ without an increase in the incidence of severe complications [22]. Recent studies have reported that the R-MPV regimen has shown excellent efficacy with CR rates of $69-79 \%$ and ORR rates of 95-96\% at the end of induction chemotherapy $[6,16]$. The present study has limitations inherent to any retrospective analysis and has no data from patients in whom HDC-ASCT was intended but not actually used. Nevertheless, a significant increase in $\mathrm{CR} / \mathrm{Cru}$ rate was observed during induction chemotherapy, increasing from $45.4 \%$ at the time of interim analysis to $81.8 \%$ prior to the initiation of HDC-ASCT.

The second point of discussion is the efficacy and tolerability of the conditioning regimen for ASCT. The outcomes of HDC-ASCT as upfront treatment in patients with PCNSL varied in a few single-arm phase 2 trials and retrospective studies (Table 4). Although no formal comparison of conditioning regimens has been conducted to date, historical results gathered after using the BEAM regimen [carmustine (BCNU), etoposide, cytarabine, and melphalan] were disappointing, with a modest treatment response rate and a 2 -year OS rate of $60 \%$ [10]. According to a previous meta-analysis conducted by Alnahhas et al. [23], BCNU/TT carried the lowest risk of TRM and had a response rate equivalent to that of TBC, while TBC achieved a lower relapse rate and numerically superior OS and PFS rates. A recent investigation by Omuro et al. [16]. found that the 2-year PFS and OS rates in their study population were $81 \%$, with a median follow-up period of 45 months. TBC is an effective conditioning regimen, but its toxicity remains a major concern that requires further evaluation. Septic complications, mostly bacterial infections, occur in one-third of treated patients, with grade 3 or greater febrile neutropenia found in $42 \%$ of patients and a TRM of up to $19 \%$ [24-26]. At our institution, patients who are young and fit for intensive therapy have been used as criteria to evaluate the readiness for TBC as a conditioning regimen as suggested by Omuro et al. [16], whereas decreased-intensity TBC and $\mathrm{Bu} / \mathrm{TT}$ regimens have been used in patients older than 60 years or 
Table 4. HDC-ASCT as upfront treatment in previous studies in PCNSL.

\begin{tabular}{|c|c|c|c|c|c|c|c|c|c|c|}
\hline Reference & $\mathrm{N}$ & $\begin{array}{l}\text { Median age } \\
\text { (range) }\end{array}$ & $\begin{array}{l}\text { Induction } \\
\text { therapy }\end{array}$ & $\begin{array}{l}\mathrm{CR} / \mathrm{ORR} \text { to } \\
\text { induction }\end{array}$ & $\begin{array}{l}\text { Transplanted } \\
\text { patients }\end{array}$ & $\begin{array}{l}\text { Conditioning } \\
\text { regimen }\end{array}$ & WBRT & OS & $\begin{array}{l}\text { Median } \\
\text { FU (mo) }\end{array}$ & TRM \\
\hline [10] & 28 & $53(25-71)$ & HD-MTX $\rightarrow$ ARAC & $29 \% / 50 \%$ & $50 \%$ & BEAM & No & 2 years: $55 \%$ & 28 & $4 \%$ \\
\hline [15] & 30 & $54(27-64)$ & $\begin{array}{l}\text { HD-MTX } \rightarrow \\
\text { ARAC }+ \text { TT }\end{array}$ & $20 \% / 70 \% \rightarrow 38 \% / 92 \%$ & $77 \%$ & $\mathrm{BCNU} / \mathrm{TT}$ & Yes & 5 years: $69 \%$ & 63 & $3 \%$ \\
\hline [11] & 25 & $52(21-60)$ & $\begin{array}{l}\mathrm{MBVP} \rightarrow \mathrm{IFO}+ \\
\mathrm{ARAC}\end{array}$ & $44 \% / 84 \%$ & $68 \%$ & BEAM & Yes & 4 years: $64 \%$ & 34 & $4 \%$ \\
\hline [13] & 23 & $55(18-69)$ & HD-MTX & $17 \% / 65 \%$ & $70 \%$ & $\mathrm{Bu} / \mathrm{TT}$ & Yes & 2 years: $48 \%$ & 15 & $13 \%$ \\
\hline [12] & 11 & $52(33-65)$ & $\mathrm{HD}-\mathrm{MTX} \rightarrow$ ARAC & $73 \% / 100 \%$ & $100 \%$ & BUCYE & Yes & 2 years: $89 \%$ & 25 & $0 \%$ \\
\hline [24] & 21 & $56(34-69)$ & $\mathrm{MPV} \rightarrow \mathrm{ARAC}$ & $24 \% / 86 \%$ & $100 \%$ & TBC & No & 5 years: $44 \%$ & 60 & $14 \%$ \\
\hline [14] & 13 & $56(35-65)$ & $\mathrm{MPV} \rightarrow \mathrm{ARAC}$ & $31 \% / 100 \%$ & $46 \%$ & LEED & Yes & 3 years: $76 \%$ & 44 & $0 \%$ \\
\hline [16] & 33 & $57(23-67)$ & R-MPV & $68 \% / 97 \%$ & $81 \%$ & TBC & No & 3 years: $81 \%$ & 45 & $12 \%$ \\
\hline
\end{tabular}

Abbreviations: ARAC, cytarabine; BEAM, carmustine, etoposide, cytarabine, and melphalan; BCNU, carmustine; Bu, busulfan; BUCYE, busulfan, cyclophosphamide, and etoposide; CR, complete response; FU, follow-up; HD-MTX, high-dose methotrexate; IFO, ifosfamide; LEED, cyclophosphamide, etoposide, melphalan, and dexamethasone; MBVP, methotrexate, carmustine, etoposide, and methylprednisolone; MPV, methotrexate, vincristine, and procarbazine; ORR, overall response rate; OS, overall survival; R-MP, MPV plus rituximab; TBC, thiotepa, busulfan, and cyclophosphamide; TRM, treatment-related mortality; TT, thiotepa; WBRT, whole-brain radiotherapy.

who are less fit. The current study reported a high response rate with a significant improvement in $\mathrm{CR} / \mathrm{CRu}$, from $81.8 \%$ before HDC-ASCT to $100 \%$ after HDC-ASCT. In addition, the 2-year PFS and OS rates were $84 \%$ and $88 \%$, respectively. In this study, the median OS and PFS rates were lower than those in the previous study by Omura et al. [16] as one patient died of pneumonia in this study, unrelated to their disease. No serious complications occurred during the treatment period or course of TRM, which is notable despite the study's major limitations, such as its small patient number and the relatively short follow-up period.

Despite the significant improvements in the management of PCNSL, nearly half of the responders will relapse, which occurs within 2 years after initial diagnosis [27, 28]. After a median follow-up period of 19.6 months, approximately $18 \%$ of patients showed relapse. Although only limited interpretation can be performed due to the small number of patients and short follow-up period, the patients who relapsed within 1 year after ASCT had poorer survival outcomes. Treatment of patients with relapsed and refractory PCNSL remains one of the major unmet clinical needs. The prognosis of R/R PCNSL is extremely poor, with a median OS of 3-5 months [29]. Hence, further clinical trial data are required to guide therapeutic management in this group of patients.

The present study has several limitations. Given the single-center, retrospective nature of this investigation, an undefined bias concerning the clinical outcomes possible exist. Interpretation of the results should be performed with caution due to the small sample size and relatively short follow-up duration; most patients (68\%) included in this study were diagnosed from 2018 onward. Our study could not verify the IELSG score for prognostication of survival. Increased CSF protein levels are associated with a poor prognosis [17, 30]. However, adopting CSF parameters as survival predictors remains a problem, given the difficulty of choosing the cutoff value to define unfavorable features. In the IELSG scoring system, the cutoff value for a normal CSF protein concentration was $45 \mathrm{mg} / \mathrm{dL}$ in patients aged 60 years or younger but $60 \mathrm{mg} / \mathrm{dL}$ in patients aged 60 years or older [17]. When these cutoff values were applied, $95 \%$ of patients showed elevated CSF protein concentrations; hence, CSF protein concentration was thought to be unsuitable as a prognostic factor for discrimination in the current study. Different laboratory methodologies account for significant discrepancies across institutions and published reference intervals [31]. In other words, the cutoff value for CSF protein concentration as a predictor of patient's prognosis cannot be applied uniformly. Hence, further studies using a well-established prognostic scoring system with a better knowledge of PCNSL, especially with the inclusion of histopathologic and molecular variables, are needed. Finally, formal neurocognitive and quality-of-life assessments were not performed.

In conclusion, R-MPV followed by thiotepa-based HDC-ASCT as a consolidation regimen resulted in good response rates and a favorable toxicity profile among patients with newly diagnosed PCNSL.

\section{ACKNOWLEDGMENTS}

The authors thank the patients who participated in this trial and their families.

\section{Authors' Disclosures of Potential Conflicts of Interest}

No potential conflicts of interest relevant to this article were reported.

\section{REFERENCES}

1. Ferreri AJ, Abrey LE, Blay JY, et al. Summary statement on 
primary central nervous system lymphomas from the Eighth International Conference on Malignant Lymphoma, Lugano, Switzerland, June 12 to 15, 2002. J Clin Oncol 2003;21:2407-14.

2. Panageas KS, Elkin EB, DeAngelis LM, Ben-Porat L, Abrey LE. Trends in survival from primary central nervous system lymphoma, 1975-1999: a population-based analysis. Cancer 2005;104:2466-72.

3. Ferreri AJ. How I treat primary CNS lymphoma. Blood 2011;118:510-22.

4. Ferreri AJ, Reni M, Foppoli M, et al. High-dose cytarabine plus high-dose methotrexate versus high-dose methotrexate alone in patients with primary CNS lymphoma: a randomised phase 2 trial. Lancet 2009;374:1512-20.

5. Rubenstein JL, Hsi ED, Johnson JL, et al. Intensive chemotherapy and immunotherapy in patients with newly diagnosed primary CNS lymphoma: CALGB 50202 (Alliance 50202). J Clin Oncol 2013;31:3061-8.

6. Morris PG, Correa DD, Yahalom J, et al. Rituximab, methotrexate, procarbazine, and vincristine followed by consolidation reduced-dose whole-brain radiotherapy and cytarabine in newly diagnosed primary CNS lymphoma: final results and long-term outcome. J Clin Oncol 2013;31:3971-9.

7. Gavrilovic IT, Hormigo A, Yahalom J, DeAngelis LM, Abrey LE. Long-term follow-up of high-dose methotrexate-based therapy with and without whole brain irradiation for newly diagnosed primary CNS lymphoma. J Clin Oncol 2006;24:4570-4.

8. DeAngelis LM, Seiferheld W, Schold SC, Fisher B, Schultz CJ; Radiation Therapy Oncology Group Study 93-10. Combination chemotherapy and radiotherapy for primary central nervous system lymphoma: Radiation Therapy Oncology Group Study 93-10. J Clin Oncol 2002;20:4643-8.

9. Correa DD, Rocco-Donovan M, DeAngelis LM, et al. Prospective cognitive follow-up in primary CNS lymphoma patients treated with chemotherapy and reduced-dose radiotherapy. J Neurooncol 2009;91:315-21.

10. Abrey LE, Moskowitz CH, Mason WP, et al. Intensive methotrexate and cytarabine followed by high-dose chemotherapy with autologous stem-cell rescue in patients with newly diagnosed primary CNS lymphoma: an intent-to-treat analysis. J Clin Oncol 2003;21:4151-6.

11. Colombat P, Lemevel A, Bertrand P, et al. High-dose chemotherapy with autologous stem cell transplantation as first-line therapy for primary CNS lymphoma in patients younger than 60 years: a multicenter phase II study of the GOELAMS group. Bone Marrow Transplant 2006;38:417-20.

12. Yoon DH, Lee DH, Choi DR, et al. Feasibility of BU, CY and etoposide (BUCYE), and auto-SCT in patients with newly diagnosed primary CNS lymphoma: a single-center experience. Bone Marrow Transplant 2011;46:105-9.

13. Montemurro M, Kiefer T, Schüler F, et al. Primary central nervous system lymphoma treated with high-dose methotrexate, highdose busulfan/thiotepa, autologous stem-cell transplantation and response-adapted whole-brain radiotherapy: results of the multicenter Ostdeutsche Studiengruppe Hamato-Onkologie OSHO-53 phase II study. Ann Oncol 2007;18:665-71.

14. Miyao K, Sakemura R, Imai K, et al. Upfront autologous stem-cell transplantation with melphalan, cyclophosphamide, etoposide, and dexamethasone (LEED) in patients with newly diagnosed primary central nervous system lymphoma. Int J Hematol 2014;100:152-8.

15. Illerhaus G, Marks R, Ihorst G, et al. High-dose chemotherapy with autologous stem-cell transplantation and hyperfractionated radiotherapy as first-line treatment of primary CNS lymphoma. J Clin Oncol 2006;24:3865-70.

16. Omuro A, Correa DD, DeAngelis LM, et al. R-MPV followed by high-dose chemotherapy with TBC and autologous stem-cell transplant for newly diagnosed primary CNS lymphoma. Blood 2015;125:1403-10.

17. Ferreri AJ, Blay JY, Reni M, et al. Prognostic scoring system for primary CNS lymphomas: the International Extranodal Lymphoma Study Group experience. J Clin Oncol 2003;21:266-72.

18. Abrey LE, Batchelor TT, Ferreri AJ, et al. Report of an international workshop to standardize baseline evaluation and response criteria for primary CNS lymphoma. J Clin Oncol 2005;23:5034-43.

19. Birnbaum T, Stadler EA, von Baumgarten L, Straube A. Rituximab significantly improves complete response rate in patients with primary CNS lymphoma. J Neurooncol 2012;109:285-91.

20. Gregory G, Arumugaswamy A, Leung T, et al. Rituximab is associated with improved survival for aggressive B cell CNS lymphoma. Neuro Oncol 2013;15:1068-73.

21. Holdhoff M, Ambady P, Abdelaziz A, et al. High-dose methotrexate with or without rituximab in newly diagnosed primary CNS lymphoma. Neurology 2014;83:235-9.

22. Ferreri AJ, Cwynarski K, Pulczynski E, et al. Chemoimmunotherapy with methotrexate, cytarabine, thiotepa, and rituximab (MATRix regimen) in patients with primary CNS lymphoma: results of the first randomisation of the International Extranodal Lymphoma Study Group-32 (IELSG32) phase 2 trial. Lancet Haematol 2016;3:e217-27.

23. Alnahhas I, Jawish M, Alsawas M, et al. Autologous stem-cell transplantation for primary central nervous system lymphoma: systematic review and meta-analysis. Clin Lymphoma Myeloma Leuk 2019;19:e129-41.

24. Alimohamed N, Daly A, Owen C, Duggan P, Stewart DA. Upfront thiotepa, busulfan, cyclophosphamide, and autologous stem cell transplantation for primary CNS lymphoma: a single centre experience. Leuk Lymphoma 2012;53:862-7.

25. Cheng T, Forsyth P, Chaudhry A, et al. High-dose thiotepa, busulfan, cyclophosphamide and ASCT without whole-brain radiotherapy for poor prognosis primary CNS lymphoma. Bone Marrow Transplant 2003;31:679-85.

26. Cote GM, Hochberg EP, Muzikansky A, et al. Autologous stem cell transplantation with thiotepa, busulfan, and cyclophosphamide (TBC) conditioning in patients with CNS involvement by non-Hodgkin lymphoma. Biol Blood Marrow Transplant 2012; 18:76-83.

27. Jahnke K, Thiel E, Martus P, et al. Relapse of primary central nervous system lymphoma: clinical features, outcome and prognostic factors. J Neurooncol 2006;80:159-65.

28. Hoang-Xuan K, Bessell E, Bromberg J, et al. Diagnosis and treatment of primary CNS lymphoma in immunocompetent patients: guidelines from the European Association for NeuroOncology. Lancet Oncol 2015;16:e322-32.

29. Langner-Lemercier S, Houillier C, Soussain C, et al. Primary CNS 
lymphoma at first relapse/progression: characteristics, management, and outcome of 256 patients from the French LOC network. Neuro Oncol 2016;18:1297-303.

30. Blay JY, Lasset $\mathrm{C}$, Carrie $\mathrm{C}$, et al. Multivariate analysis of prognostic factors in patients with non HIV-related primary cerebral lymphoma. A proposal for a prognostic scoring. Br J Cancer 1993;
67:1136-41

31. Breiner A, Moher D, Brooks J, et al. Adult CSF total protein upper reference limits should be age-partitioned and significantly higher than 0.45 g/L: a systematic review. J Neurol 2019;266: 616-24. 